\title{
NUEVAS OBSERVACIONES DE CRONO-PALEOGRAFÍA IBÉRICA LEVANTINA
}

POR

\author{
JESÚS RODRÍGUEZ RAMOS
}

Universitat Autònoma de Barcelona

PALABRAS CLAVE: Epigrafía ibérica. Escritura ibérica levantina. Paleografía. Siglos V-I a.C. / I d.C.

KEY WORDS: Iberian epigraphy. Iberian Levantine writing. Paleography. $5^{\text {th }}-1^{\text {st }} \mathrm{BC} / 1^{\text {st }}$ centuries AD.

\section{RESUMEN}

Este artículo supone la continuación de nuestro primer estudio sobre la cronología paleográfica de la escritura ibérica levantina. Se añaden al sistema dos signos (bi y bo), así como se comentan algunas variantes menores de otros signos. Se revisa además la nueva documentación sobre te-1. Finalmente se efectúa una breve descripción de la distribución de las principales zonas que se distinguen por tener variantes de signos propias.

\section{SUMMARY}

This paper is the continuation of a previous study of the chronological palaeography of Iberian Levantine writing. Two signs are added to the system ( $b i$ and $b o$ ) and comments are made on some minor variations of other signs. The new documentation of $t e-1$ is also revised. Finally, the distribution of the main areas demarked by variations of the signs themselves is briefly described.

Este artículo constituye la continuación de mi primera exposición del método crono-paleográfico aplicado a la escritura ibérica levantina. Los objetivos de esta continuación son cuatro: 1) La incorporación al análisis completo de dos nuevos signos ( $b i$ y $b o^{1}$ ); 2) el examen de algunas formas peculiares de algunos signos (formas de $b u$ y de $e$ ); 3) las novedades sobre la cronología de $t e-1$; y 4) revisión de las zonas geográficas que utilizan signarios distintivos y de las zonas arcaizantes.

La problemática abordada es más compleja que la del anterior trabajo dado que se tratan aspectos para los que hay menos datos y cuya clasificación es más difícil. También hay que tener en cuenta que, aunque abundan las noticias de nuevas inscripciones, son pocas las que han sido correctamente publicadas o puestas a disposición de los investigadores, lo cual limita el caudal de nueva información a pesar de los años transcurridos. Por todo ello, el

\footnotetext{
1 Por condicionantes tipográficos se sustituye la notación ortodoxa de la transcripción de los signos nativos en negrita por cursiva.
}

presente artículo no pretende más que aportar unas cuantas anotaciones útiles.

Cabe recordar previamente que las fechas de inicio y fin de uso de la escritura son arbitrarias. Seguramente existen inscripciones previas al 400 a.C. y se documentan claramente algunas posteriores al 50 a.C. como la bilingüe de Manises- $01^{2}$ sobre una terra sigillata sudgálica que se adentraría en el s. I d.C. ${ }^{3}$.

\section{LA CRONO-PALEOGRAFÍA DE LOS SIGNOS $b i$ Y bo}

La problemática de estos dos signos es bien distinta. El signo bo es de fácil definición morfológica, pero las formas minoritarias están escasamente documentadas; por el contrario el signo bi, abundantemente documentado, presenta grandes dificultades en su reconocimiento tipológico. Ello es debido a que su extensión en forma de gancho es susceptible de recibir un trazado descuidado y, así, en inscripciones largas no es raro encontrar dos o tres formas distintas, pese a que suela predominar una. Por ello la tipología de $b i$ debe reducirse a unos cuantos prototipos de rasgos bien diferenciados y, aun así, no puede descartarse la posibidad de intrusiones accidentales por un error caligráfico.

\section{El signo bo}

Podemos distinguir cuatro formas, tres de lectura segura y una cuarta en que no puede aún descartarse por completo la lectura alternativa $t a$.

bo-1. Aspa con un trazo secante vertical. Éste puede ser efectivamente uno (bo-la) o ser dos, un apéndice superior y otro inferior $(b o-1 b)$. No descar-

2 Sigo la numeración de la base de datos paleográfica de Rodríguez Ramos (en prensa 1). Las equivalencias y referencias completas se encuentran en el apéndice que va tras el artículo

3 Pero no otras piezas como el sello ibérico de Azaila (E.1.287) que Panosa (1996: 22) identifica como terra sigillata apuntando una datación de s. I d.C. De hecho es un mortero con decoración de dediles (Beltrán 1990: 216) probablemente de ca. 100 a.C. Azaila es un yacimiento destruido durante las guerras sertorianas y, consecuentemente, carece de terra sigillata. 
Cunadro 1

\begin{tabular}{|c|c|c|c|c|c|c|c|}
\hline \multicolumn{8}{|c|}{ 1. Formas del signo bi: } \\
\hline bi-1a & $\Gamma$ & bi-1b & $\Gamma \Gamma$ & bi-1c & $\Gamma$ & & \\
\hline $\mathrm{bi}-2 \mathrm{a}$ & $\Gamma$ & $\mathrm{bi}-2 \mathrm{~b}$ & $\Gamma$ & & & & \\
\hline bi-3 & $P P$ & bi-4 & $\Gamma$ & & & & \\
\hline bi-5a & $P$ & bi- $5 \mathrm{~b}$ & $P$ & bi- $-5 c$ & $P \Gamma$ & bi-5d & $P$ \\
\hline \multirow[t]{2}{*}{ bi-6 } & $\Gamma$ & & $\rho$ & bi- 8 & $\Gamma$ & & \\
\hline & \multicolumn{7}{|c|}{ 2. Formas del signo bo: } \\
\hline \multirow[t]{2}{*}{ bo-1 } & X $X$ & & 米 & bo-3 & 天 & bo-4 & $X X$ \\
\hline & \multicolumn{7}{|c|}{ 3. Formas del signo $b u$ : } \\
\hline bu-1 & $\square$ & bu-2 & 目 & bu-3 & 目 & bu-4 & 四 \\
\hline \multicolumn{8}{|c|}{ 4. Signos de la inscripción Can Jordi-01: } \\
\hline & I $N^{2}$ & & ^ & & $Y<$ & & \\
\hline
\end{tabular}

to que $b o-1 a$ sea más moderno que $b o-1 b$ pero los datos de los que dispongo no permiten distinguir entre ambas variantes.

Ésta parece ser la forma originaria del signo y está atestiguada en inscripciones del s. IV (Ensérune-03, Ampurias-24, Ullastret-13). En los yacimientos del sur de Francia no se encuentran ni bo-2 ni $b o-3$ por lo que, al ser tan extraño el posible $b o-4$, indica que es prácticamente el único signo bo usado en la zona. Así comprobamos que se encuentra en inscripciones tardías de Ensérune: Ensérune-336, Ensérune-337 (ambas de 150-50 a.C.) y Ensérune61 (probablemente del s. II ya avanzado). Este uso podría llegar hasta Ampurias, donde el plomo Ampurias-06 parece posterior al 150 . Se encuentra asimismo en una emisión monetal de la ceca untikesken (CNH: 144, n. ${ }^{\circ}$ 22) por más que no puede descartarse que, como concluye Villaronga, no se trate en realidad de una marca numeral.

Sin embargo, al sur de esta zona las cosas no son tan sencillas. De las 17 inscripciones en que se encuentra, 16 si eliminamos la de Azuara-01 que es una marca numeral, sólo 2 admiten una datación iberorromana como posteriores al 180/175 a.C. Está bien documentado en textos de la fase transicional (ca. 200) como el plomo Bernabé-1, las inscripciones de Liria-03, 16 y 27, o en anteriores como el plomo de Palamós-01 o la pátera de Tivissa-01.

Los dos testimonios posteriores son los grafitos cerámicos de Azaila-287 (técnicamente 200-75, pero probablemente 150-75) y sobre todo el de Oliete-01 (100-65). Se trata de la zona de Teruel donde el alfabeto ibérico está en contacto con el celtibérico tipo Luzaga (que sí conserva la forma de bo- $l$ para su lengua) y es una zona arcaizante. Incluso así, ha de notarse que en Azaila es el único de 14 casos en que está presente esta forma. Otro testimonio tardío de $b o-1$ debe de ser la leyenda monetal bon en la ceca bolśken (CNH: 210-212, pieza n. 3 de la segunda mitad del s. II), donde sin embargo para el nombre de la ciudad usan bo-3. Esta observación, uso de dos tipos de bo en una misma moneda, constituye un apoyo a la teoría de Villaronga de que la marca bon no sea en realidad fonética, sino una marca de valor correspondiente a 'un denario'. Ello no obstante, no puede estarse seguro, dado que si bien el signo bo- 1 coincide con la marca romana para denario, posteriormente se usa también bo-3 para bon e incluso, en paralelo con el paso de la ceca a olśken, se encuentra on. Pero debemos recordar que esta moneda corresponde al grupo pirenaico, cuya adscripción a lo ibérico no es segura, que muestra conexiones con grafías celtibéricas (el propio signo $k e$ ) y que, en definitiva, es otra zona arcaizante ${ }^{4}$.

Consecuentemente bo- 1 es un signo de forma clásica en la zona al norte de Ampurias (o más prudentemente Gerona y sur de Francia), es un signo arcaizante en la zona de Teruel y aun así poco frecuente) y probablemente inexistente, al ser substituido por $b o-3$ en el resto de la zona ibérica con posterioridad a los inicios del s. II, siendo un indicio de datación pre $180^{5}$.

bo-2: es la forma de asterisco, un aspa sobre una cruz, correspondiente, en principio, a un desdoblamiento del signo bo en el sistema de notación dual de oclusivas. Pero esto no deja de presentar problemas.

Hace tiempo que se ha observado el problema de que, si bien con dentales y velares este desdoblamiento corresponde a lo que en greco-ibérico es la distinción entre sordas y sonoras ( $\mathrm{t} / \mathrm{d}, \mathrm{k} / \mathrm{g}$ ), en íbero sólo se conoce el fonema /b/, pero no /p/ y es bo el único de los silabogramas de oclusiva labial que se

4 Obsérvese que una serie de cecas comparten esta marca bon / on : sesars (CNH: 209) con bon; iaka (CNH: 215) bon; sekia (CNH: 215) tiene on; arsaos (CNH: 252) on; arsakos ( $C N H$ : 256) on; bentian (CNH: 257) on. Si no es una marca de valor debiera ser una inusitada marca de pertenencia regional.

5 Hay que reconocer el problema que representa este signo cuando aparece aislado o junto a una barra vertical. No siempre puede asegurarse que sea $b o-1$ y no $b o-2$ ni puede descartarse que se trate de una marca ágrafa pues como tal es usada en la epigrafía griega y romana ocasionalmente. 
desdobla ${ }^{6}$. Pero por otra parte esta variante está ausente del sur de Francia y sólo se encuentra sobre ocho inscripciones (considerando los tres plomos de la tumpa de Orleyl un único documento). Se encuentra en el plomo de Ullastret-03 y en el de Ampurias-23, pero las demás ocurrencias son todas de la zona edetana (Liria, Bernabé, Villares-02, Orleyl y Castellón). Todas estas inscripciones serían de los siglos IV y II a.C. salvo Liria-03 y 59 y Bernabé-01 que tanto podrían ser del s. III como adentrarse en los inicios del s. II hasta el 190 ó 180 . Puede concluirse que este signo desaparece hacia el 200/180.

La extraña distribución del mismo plantea nuevos interrogantes. El signo, como hemos indicado, aparenta corresponder al desdoblamiento de oclusivas del sistema dual. Sin embargo esto sólo es válido para el plomo de Ullastret y el de Ampurias, puesto que, según opino actualmente, este sistema sólo se usa al norte del Ebro. A partir de aquí, las realizaciones edetanas con rasgos extra carecen actualmente de explicación, pero ¿qué sucede con la gran cantidad de documentos que sí usan sistema dual en el sur de Francia?

Existen dos posibles soluciones, ninguna de ambas plenamente satisfactoria. La primera, minimalista, indicaría que no aparece bo-2 porque, como se ha señalado, no se desdobla la oclusiva labial y el signo no hace falta alguna; su ocurrencia en Gerona sería alguna solución epicórica. La otra solución sería suponer que el desdoblamiento de bo sí se ha efectuado, pero bajo otra forma. Desde ese punto de vista es muy a tener en cuenta que, ya para los plomos de Pech-Maho, Solier (1979: 73) propuso la lectura de variante de $b o$ a $x-4$, basándose en la sugerente reconstrucción de un onomástico galo botuorís. La extraña distribución de bo-2 es, desde luego, un argumento a favor de la propuesta de Solier y es posible que sea la solución correcta, pero restan aún algunos problemas por solventar.

Para la ceca pirenaica $s$-[x-4]-sars (comúnmente leída sesars, $C N H$ : 209) hay que suponer que es una homomorfia casual y que no son el mismo signo. Sin embargo, hay otro signo $(x-3)$ cuya excepcionalidad y similitud con $x$ - 4 recomiendan considerar a ambos como un mismo signo. Aquí el primer problema radica en que se conoce esta forma ya en el plomo de Palamós. Éste es sensiblemente anterior a los de Pech-Maho y hace probable (aunque no for-

${ }^{6}$ En principio, la hipótesis alternativa por defecto sería suponer una aspiración de la /b/ ante vocal de timbre posterior. Indicios de la misma los tenemos en la infrecuencia del signo $b u$ en ibérico (salvo en el sur de Francia donde parece una variante dialectal de bo), en evoluciones del tipo bolśken $>$ olśken / Osca, o en la ceca neŕonken ( $\mathrm{CNH}$ : 437) correspondiente a Narbo. zoso) el que la forma $x$ - 4 derive de $x$-3. Resulta pues que si la forma $x-4$ guarda un notable parecido con $b o$, que permitiría explicar su derivación aunque no su valor fonético, este parecido es mucho menor en lo concerniente a $x-3$. Otro problema es que $x$-3 parece encontrarse como sufijo de onomástico en Osséja-2 (al menos en lo que deja ver la pésima caligrafía de estas inscripciones rupestres) y nos faltan paralelos de un sufijo adonomástico bo.

bo-2: 400/350-200/180.

bo-3: En esta variante el trazo secante sobre el aspa pasa a ser horizontal en vez de vertical y muestra una tendencia a reducirse en longitud. Su distribución y cronologías son coherentes pero cabe destacar que la cardinalidad de sus 21 ocurrencias es engañosa, ya que 13 son de Azaila. Nos hemos de limitar a ocho yacimientos.

Los dos más septentrionales son Palamós-02 y Gruissan-01. Sin embargo técnicamente esto se reduce a Palamós, dado que el plomo de Gruissan-01 presenta una problemática especial al proceder de un barco naufragado. Paleográficamente puede proceder de Gerona y no del sur de Francia. El límite meridional lo ponen Sagunto y Montaña Frontera.

La gran presencia de esta variante en Azaila nos asegura la cronología de la fase 100-75, coherente con el naufragio de Gruissan (100-65), el ánfora de Els Monjos-02 (125-50) y el plomo de Yátova (paleográficamente del estilo Iberorromano II, 150-50). Sólo hay dos inscripciones que, paleográficamente, puedan remontarse algo en antigüedad; ambos por presentar $s$-3 signo que en principio desaparecería hacia el 175/150. Una es el plomo de Sagunto-29 que al presentar $a-5 a$ y $s-3$ debe de corresponder al lapso $225-175 / 150^{7}$. Otra es Palamós-02 que al presentar $a-4,-2, s-3$ y be-7 debe de corresponder al lapso 190-150.

Dada la ausencia de bo-3 en inscripciones de estilo transicional el post quem probable es de 180/ 175 , lo que enfatiza la idea de que la forma $s$ - 3 persiste en su uso hacia el segundo cuarto del s. II.

bo-3: 180-50 (no usado en el sur de Francia).

bo-4: Este signo lo había clasificado anteriormente como ta-2 y se encuentra en dos inscripciones del sur de Francia: Ensérune-16 (325-225) y Elne-11(225-180). Podemos suponer que esta forma tuvo algún uso hacia la segunda mitad del s. III. Paleográficamente considero más probable que se trate de bo dado que corresponde a una evolución de

7 En la parte superior hay un signo fragmentado que parece $b o-1$. Como, de ser efectivamente así, sería el único caso de coexistencia, puede plantearse, más que corresponda a una fase de transición o que el plomo sea falso, que se trate de un numeral. 
$b o-1 b$ en el que los dos trazos del segmento vertical de $b o$, al ser independientes, se han ido lateralizando. Por otra parte no se entiende bien la necesidad de dos trazos extras para desdoblar el signo ta, en vez de uno. Sin embargo esta interpretación sólo es probable.

\section{El signo bi}

Altamente problemático por la dificultad en distinguir lo que es un mal trazado de lo que era el signo ideal del signario que se utilizaba. Para este signo tendremos en cuenta cuatro trazos y dos ángulos. El primer trazo es el típicamente vertical y largo. El segundo trazo parte de la parte superior del primero y transversalmente se dirige en dirección progresiva de la escritura. Entre el primer y el segundo trazo tenemos el primer ángulo. El segundo trazo surge del extremo del segundo y se dirige hacia abajo, tanto en vertical como en dirección contraria al sentido de la escritura. Entre el segundo y el tercer trazo tenemos el segundo ángulo. El cuarto trazo surge eventualemnte del tercero y suele implicar cierto sentido de espiral. Dicho esto, mi propuesta actual de clasificación queda de la siguiente manera:

bi-1: signo de tres trazos en ángulo recto en el que el tercer trazo no es minúsculo. Se admite también $(b i-1 b)$ aquél en el que la inclinación del segundo ángulo entre el segundo y el tercer trazo es algo menor de noventa grados. De bi- $1 a$ tenemos 10 ocurrencias de las que hay varias posteriores al 150 (Solsona-03, plomo de Yátova, Montlaurés-03, Azaila-369, ésta post 100). Poco decisorias son las dataciones de Ensérune 268 y 302 (200-50) y poco clara la base de una especie de crátera Ensérune-26 que paleográficamente ha de ser posterior al 225 . Una datación intermedia la tenemos en el dipinti de Vielle-Toulouse-3 (170-130). Es pues claramente un signo iberorromano y, aunque pueda ser más tardío, parece probable asimilarlo a las innovaciones de ca. $180 / 175$.

Obsérvese que excluyo de la categoría de $b i-l$ una serie de inscripciones, siempre sobre cerámica ática, que en ocasiones han sido interpretadas como ibéricas pero que no son más que numerales y marcas comerciales griegas ${ }^{8}$.

Por su parte la forma $b i-1 b$ presenta sólo cinco ocurrencias, pero muy similares a las de bi-la: $d i$ -

${ }^{8}$ Así lo que se lee $d u b i$ III no es más que el numeral 18 $(\Delta \Pi I I I)$. En otras ocasiones la p es abreviatura de $\pi$ olkí $\lambda$ o $\varsigma$. El signo que Untermann ha interpretado como nexo bir sería el numeral 50 pinti de Vielle-Toulouse, plomo de Yátova, Azaila320 y dos inscripciones de Ensérune de los ss. II-I. $b i-1 a$ y $b i-1 b: 180-50$.

La forma $b i-1 c$ corresponde a aquélla en el que el tercer trazo es diminuto pero con un segundo ángulo recto. Se esperaría una cronología también tardía, pero de sus tres casos registrados uno es una cerámica ática de Montlaurés-01 (aunque Untermann no refiere más que el dibujo de sus dos signos). Por ello, en principio, parece más prudente abstenerse de darle ninguna cronología.

$b i-2$ : Corresponde a la forma en el que el segundo ángulo es cerrado. La variante 1a posee el tercer trazo de un tamaño normal, mientras que la $1 \mathrm{~b}$ corresponde a su realización diminuta.

La forma $b i-2 a$ es clásica, apareciendo en todas las épocas bien documentada. El segmento inferior está probado por los casos en Azaila, especialmente Azaila-318 (100-75), así como el mosaico de Andelos (150-50, ca 100). Previa al 190 ha de ser Ullastret-08. Se encuentra en el plomo de Pech-Maho-38 (250-200, probablemente fines del s. III), mientras que del s. IV son Ampurias-12 y Pech-Maho-02. En todo caso, llama la atención su no asociación a los abundantes plomos con estilos paleográficos transicionales.

Es difícil dar una datación a la forma $2 \mathrm{~b}$, dado que si bien sus dos ocurrencias son tardías, ss. II-I, se trata sólo de dos casos.

$b i$-3: En esta forma tanto el primer como el segundo ángulo son cerrados, aunque en ocasiones los trazos segundo y tercero se efectúan como una curvatura. Esta forma también es clásica. En el s. Iv tenemos Ensérune-03; en el III, Palamós-01 y los plomos de Pech-Maho 34-36. En fase tardía sólo se documenta con claridad en Azaila (9 casos) y en Oliete-08. Esto podría hacer suponer que es una forma arcaizante y, sí, ciertamente es poco frecuente en inscripciones tardías costeras, pero una forma asimilable se encuentra en el plomo de Yátova-03, por lo que, como mucho, es un débil indicio de arcaísmo.

bi-4: Forma poco documentada pero de forma muy coherente. Se encuentra en los plomos de Pech-Maho 34-36 (225-200), en Ensérune-124 (225-50), en Can-Rodón-02 (250-200) ${ }^{9}$ y el plomo de Ampurias-23 (225-200). Todos estos datos señalan a un uso epicórico (Gerona-sur de Francia) en el último cuarto del s. III. Esta fecha además es coherente con el hecho de que esta forma ya no se confundía con $l$ al haberse creado precisamente hacia el 225 , la forma 'lambdamorfa' $l-2$.

bi-4: 225-200, ¿tal vez también inicios del s. II?

\footnotetext{
${ }^{9}$ Corrijo aquí la lectura como biurútia.
} 
bi-5: Son las formas con gancho interno donde se encuentra incluso un cuarto trazo tendiendo a hacer una espiral de cierre. La forma ideal es la $b i-5 a$ en que el ángulo primero es cerrado, es decir, el segundo trazo es descendente y el gancho anterior, aunque formado con una única línea curvada, está bien marcado. En $b i-5 b$ se incluyen formas muy similares, pero en las que el gancho está poco desarrollado, tal vez por no terminarse el signo. En la forma $b i-5 c$ se recogen signos con el gancho interno, aunque a veces poco desarrollado, pero en las que el primer ángulo es prácticamente recto, es decir, el segundo trazo es horizontal o apenas desciende; en éstos hay tanto formas angulosas como curvadas, dado que la escasez de las primeras no permite establecer subgrupos. Finalmente, la forma $b i-5 d$ recoge formas con los cuatro trazos pero que más que en forma de gancho lo que se observa es una especie de $P$ latina arcaica, ocupando el bucle superior normalmente poco espacio respecto al conjunto del signo. El problema con esta variante es que no siempre es evidente su distinción respecto a $b i-3$.

La forma $b i-5 a$, con su característica apariencia násica, está muy bien representada. Las formas de un trazo más desarrollado son típicamente edetanas pero formas asimilables se pueden encontrar también en Cataluña y el sur de Francia. Está atestiguada en el s. IV en Ullastret-03 y 23, en Sagunto-26 y, con una forma asimilable, en el plomo de Penya del Moro-01. Del s. III han de ser los plomos de Villares (01 y 02) y de Orleyl (05 y 07). Sus últimas apariciones las hace en documentos de la fase transicional (220-180): Liria-03, Orleyl-01, Por_Granada-01*, Castellón-01 y El Solaig-01. Se encuentra una forma similar, intermedia entre la 5 a y la $5 \mathrm{c}$, aunque usándose junto a $b i-3$ en el plomo Por_Tarragona-02, cuyo lapso de realización, según su paleografía, es algo más amplio (225/200-150), pero compatible.

De la forma $b i-5 b$ sólo puedo documentar dos casos. Uno es el mismo plomo Por_Granada-01 en el que ha de ser un mal trazo de $b i-5 a$, el otro es una cerámica ática de Ullastret. Parece pues que no hay diferencia entre ambas variantes.

bi-5a: 400-180.

Las formas de $b i-5 c$ no son muy homogéneas y sí están mal representadas. De ocho casos, seis serían compatibles con la datación de $b i-5 a$. Sin embargo tenemos la forma de la estela de Cabanes-01, extremadamente angulosa, y la de la fusayola de

* Nota del editor: El signo «Por_» usado por el autor equivale a «Provincia de», esto es, procedencia desconocida dentro de esa provincia.
Oliete-06, de estilo curvo. Bien es cierto que la cronología para la primera es sólo de post 200 , que strictu sensu sería compatible con un pre 180 , pero es más probable que sea más moderna. También Oliete-06, datable a inicios del s. I, podría considerarse de una área arcaizante, pero es poco satisfactorio. Concluyamos pues, prudentemente y a la espera de nuevos testimonios, que, aunque la forma $b i-5 c$ es un indicio de arcaísmo o antigüedad, no es en absoluto una prueba.

Más claras son las conclusiones respecto a las ocho ocurrencias de $b i-5 d$. Tenemos un caso claro del s. IV (Ampurias-24) y varias inscripciones que son pre 190/180. La única que pudiera ser algo posterior es la estela de Binéfar-02, aunque no es seguro que su $b i$ no sea en realidad un bi-3. De todas formas su paleografía es arcaizante (forma compleja de $o$, forma $s-3$, dos tipos de $k i$, forma de $b e-7$ de aspecto arcaico) por lo que, aun siendo su ante quem teórico de pre 150 , lo más probable es que se realizase en el primer cuarto del s. II. Por lo tanto la cronología de uso de esta forma puede considerarse de 400/350-175, pero siempre teniendo en cuenta que su parecido con $b i-3$ crea problemas prácticos de clasificación.

bi-5c: 400/350-175.

bi-6: Forma en que el segundo trazo desciende, pero el tercero tiende a la vertical. Este signo suele ser de ductus más curvado que anguloso, dándole también un aspecto de $\mathrm{P}$ primitiva. De todas formas sus testimonios son demasiado escasos para dar una estimación cronológica, máxime cuando en el plomo de Palamós-01 coexiste con $b i-3$ por lo que puede ser producto de un mal trazado.

bi-7: Variante en forma de bucle. Ni siquiera el primer trazo es una línea recta vertical, sino que todos juntos componen una espiral curvada. Sólo tres casos documentados cuyo uso parece centrarse en 250-200/175, pero es muy poca evidencia.

bi-8: Forma en que el signo apenas está esbozado por una curva. También está muy mal documentado, por más que sus dos casos correspondan al s. III.

\section{LAS FORMAS ESPECIALES DE $b u$ Y DE $e$}

\section{Las formas de $\boldsymbol{e}$}

Respecto al signo $e$ debemos distinguir claramente las formas con dos apéndices transversales ascendentes, que sería clásica o una innovación temprana (puesto que parece rara en el s. IV y depende del testimonio de la problemática cerámica 
ática de Sidamunt-01 ${ }^{10}$ ), de aquellas formas complejas que poseen más, en especial la frecuente con tres. Mi opinión es que el último periodo de uso de las complejas sería el transicional, previo al 180/ 175; de la misma manera que no descarto que la inscripción de Sidamunt sea muy posterior a su soporte. Pero todo esto, si bien es válido como indicio, todavía no puede probarse.

Aparece muy raramente el signo $e$ de tres trazos en Azaila, aunque en posiciones donde puede ser un símbolo, como la espiga, y puede tratarse de una influencia del signario celtíbero tipo Luzaga que conserva esta forma. También se encuentra en el plomo de Ampurias-06 que probablemente es posterior al 150. Pero, aparte de la no total seguridad de la datación, coexiste con la forma simple de $e$ (un caso de cada) y creo que su $e$ de tres trazos puede ser un simple espejismo y deberse a un trazo accidental; puesto que ciertamente su tercer trazo es de aspecto irregular.

De igual manera puede localizarse en unas pocas estelas funerarias, pero que paleográficamente parecen ser de las más antiguas. Sin embargo, el problema principal lo plantea la campaniense B de Benidorm-01 donde en el breve texto kuleśtileis la primera $e$ tiene tres trazos y la segunda sólo dos. Ahora bien, el tercer trazo, el superior, de la primera $e$ tiene una inclinación diferente y tal vez una diferente profundidad y parece coincidir en inclinación con un esbozo de trazo que se observa cruzando los otros dos. Por ello no me parece imposible que primero se grabase la $e$ con el trazo superior y el poco claro seccional, pero, que al quedar demasiado horizontales respecto al sentido del texto, se corrigiesen con dos más profundos y bien orientados. Sin embargo, esto no deja de ser una mera posibilidad, por lo que es probable, pero no seguro, que las formas de $e$ complejas desapareciesen antes del 175, lo que como indicio suele dar buenos resultados. De la misma manera, es probable que la $e$ de dos trazos no sea antigua, sino que apareciese en algún momento del s. III, pero tampoco es concluyente.

\section{Las formas de bu}

De este signo podemos hacer una clasificación provisional en la que la forma $b u-1$ correspondería

${ }^{10} \mathrm{Si}$ su último signo no es una $r$ invertida (para lo cual hay precedentes) la forma de $a$ sería posterior al 225. Puede cotejarse con la interpretación de Cura (1993) para quien la escritura ibérica no llegaría al interior hasta el s. II (aunque yo no encuentro inconveniente para subirlo a finales del s. III), pudiendo corresponder las piezas previas a recomercializaciones de cerámicas ya inscritas en la costa. al rectángulo simple; la $b u-2$, al que incluye un punteado interior; $b u-3$, con una línea horizontal interior y puntos en los cuadrados internos resultantes; mientras que $b u-4$, anteriormente leída te y clasificada por mí como te- 0 , sería el rectángulo con una línea vertical interior.

El signo $b u$ es, al parecer por motivos de fonética ibérica, muy escaso en las inscripciones. Sólo tiene una cierta frecuencia en inscripciones del sur de Francia, en las que, en un aparente fenómeno dialectal, el formante de compuestos de tipo onomástico ibérico -boŕ se encuentra escrito -buŕ. El signo originario ha de ser el rectángulo básico, puesto que coincide con el bo sudlusitano y con el signo bo o $b u$ del ibérico meridional. Por otra parte sus otras variantes sólo se encuentran en Francia.

La forma $b u-2$ se encuentra en cuatro inscripciones (tres en Ensérune y una en Mailhac) que no permiten ninguna precisión cronológica. Mientras que la forma $b u-3$ es la propia de los tres primeros plomos de Pech-Maho (34-36) que son de finales del s. III.

En cambio la forma sobre la que quiero llamar la atención es la nueva bu-4 que, anteriormente, siguiendo a Untermann, había leído como te y clasificado en mi paleografía como te- 0 . Veamos sus ocurrencias:

Ensérune-21 (B.1.24) selkibuŕar (datable en

Ensérune-23 (B.1.26) lectura (325/275- 225) no clara

Ensérune-271 (B.1.275) ]busuŕ (400/300-175) signo $b u \mathrm{du}-$ doso

Ensérune-285 (B.1.289) ]ibu (??)

Como puede verse, el único segmento conspicuo es el de Ensérune-21, constituido por una marca de propiedad de nombre propio seguido de sufijo -ar. Si observamos las formas paleográficas de te (Rodríguez Ramos 1997a) vemos que las formas primitivas son redondas, mientras que las formas rectangulares son típicamente iberorromanas y que incluso así no se parecen a este signo. Es por tanto paleográficamente dudoso que exista una forma rectangular de te en pleno s. III. Pero la prueba definitiva la tenemos en el nombre propio selkibuŕ en el que el primer elemento es claramente selki (MLH III $\left.n .^{\circ} 101\right)$, mientras que para el segundo elemento Untermann recurre a interpretarlo como haplología de ete / eter / eten (MLH III n. ${ }^{\circ}$ 54). Aquí el problema no es sólo la haplología y el que en un formante que alterna $\mathrm{n} / \mathrm{r}$ sea extraña la vibrante $r$, sino inclu- 
so el que el formante eter es de los que sólo se documentan en primera posición de compuesto salvo, supuestamente, en Ensérune-21. En cambio, la lectura selkibuŕ permite identificar el segundo elemento con el formante boŕ (MLH III: 46). Éste aparece en ambas posiciones, aunque es más frecuente precisamente en la segunda, y su variante buŕ está atestiguada precisamente en el sur de Francia, en los plomos de Pech-Maho (kuleś-buŕ, atin-buŕ). Es, por tanto, seguro que ha de ser leído $b u$.

\section{CUESTIONES SOBRE LA CRONOLOGÍA DE $t e-1$}

En este apartado me centraré sobre una interesante inscripción de la que no había tenido noticia en mi anterior trabajo paleográfico y en la que parece encontrarse una forma $t e-1$ en pleno periodo iberorromano, seguramente en el tercer cuarto del s. II, y para la que no parece poder recurrirse a que provenga de una zona arcaizante. Se trata de la inscripción pintada sobre un borde de cálato procedente de Can Jordi.

Este fragmento de borde de cálato apareció en un silo rellenado, según sus excavadores hacia el 140-130 a.C., centrándolo en el tercer cuarto de siglo (Prevosti et alii 1995: 143-162; unidad estratigráfica 1004). La datación es bastante clara y se basa en la ausencia de cerámica campaniense B, frente a la presencia de campaniense A tardía. Puede destacarse la aparición de formas Lamboglia 36/ Morel 1312j (datadas por Morel en 120-100 a.C.) y Lamboglia 27 variantes Morel 2825 y $2648 \mathrm{c} 1$ (datadas ambas por Morel en 150-125), así como ánfora itálica Dressel 1A que da un post 135. Menos concluyentes son formas que, según los excavadores son sólo similares pero no idénticas a las formas Morel 2646a1 y 2914c1, cuyos modelos corresponderían a finales del s. III. Resta el problema de considerar el periodo entre la fabricación y la amortización de los materiales, escaso para el ánfora, algo mayor para la cerámica campaniense, pero poco claro para los tres cálatos pintados que se encuentran en la U.E. 1004. Es de suponer que una pieza tan elaborada tendiera a perdurar bastantes años, pero no puede descartarse tampoco una amortización ritual ". En todo caso, el momento de fabricación del cálato, que es también el momento de realización de la inscripción, parece corresponder al tercer cuarto

"Documentación a favor del uso religioso de los cálatos, así como probablemente sus inscripciones, en Rodríguez Ramos 1994. del s. II. Sería posible una fecha algo más antigua, pero es menos probable y, como veremos, la paleografía apoya la primera datación.

Respecto a la pieza en sí, Panosa (en Prevosti et alii 1995: 162) opina que «Cal excloure que el vas hagués estat dotat de text -i per tant de la decoracióen el lloc on fou trobat. Més aviat hem d'optar per 1'alternativa força probable que el vas provingui del País Valencià, concretament de Sant Miquel de Llíria, únic lloc on l'escriptura es presenta en aquesta modalitat de suport». Sin embargo, esta argumentación no es correcta: ni la tipología del cálato se parece en absoluto a los cálatos valencianos, ni éste es el único lugar donde se encuentran cálatos con inscripción, puesto que hace tiempo que se conocen los de El Castelillo de Alloza en Teruel ${ }^{12}$. Tampoco hay que olvidar que se encontró con otros dos cálatos de características de producción similar, por lo que un paquete tan homogéneo es poco probable que provenga de lejos; ni que la gran época de la cerámica pintada de Liria se acaba hacia el 180 a.C. Aunque no se pueda descartar por completo que algunas piezas fuesen posteriores, no hay una documentación clara al respecto.

Afortunadamente, contamos con un estudio minucioso de las tipologías de los cálatos ibéricos (Conde 1991 y 1992). Sin embargo, las características morfológicas de este cálato son, asímismo, excepcionales. Según la descripción, los tres cálatos de la U.E. 1004 presentan una pintura de muy mala calidad, presentando los tres decoraciones en el borde en forma de 'dientes de lobo' (uno de ellos junto con series de ondulaciones de $\mathrm{S}$ horizontales). Uno de los cálatos tiene un diámetro de borde de $25 \mathrm{~cm}$, otro de $29 \mathrm{~cm}$, y el diámetro interior del de la inscripción, de acuerdo con el dibujo, es de unos 33 $\mathrm{cm}$, pero 40 de diámetro externo. Éste presenta asas bífidas que, siempre según el dibujo proporcionado, sólo conectan con el cálato en sus extremos, característica muy poco frecuente. Aunque lo fragmentario de lo conservado no permite apreciarlo bien, parece estar separada la decoración por bandas tipo cabellera verticales y presentar una estrella-asterisco bajo el asa.

Es difícil compatibilizar estas características con los cálatos valencianos, por ejemplo con el tipo C de Conde, que tiene un diámetro de entre 9 y $20 \mathrm{~cm}$

12 El que estos cálatos sean similares en decoración a los de Liria y que sean substancialmente posteriores, me induce a pensar que corresponden a influencias de inmigrantes edetanos en la zona de Teruel, de la misma manera que explico el origen de la primera escritura celtibérica; pero son productos locales, con una tipología del soporte bien diferenciada de los valencianos. 
y nunca presenta asas de ninguna clase. Bonet (1995: 411s) expresamente indica que todos los cálatos de Liria se enmarcan perfectamente en la categoría $\mathrm{C}$ de Conde, que nunca lleva asas, que su producción se acaba a inicios del s. II, y que sólo hay una pieza de tamaño excepcional (fig. 67, pp. 142 y 144) que llega hasta los $31 \mathrm{~cm}$ de diámetro.

Algo más asimilables serían los cálatos de Teruel, cuya clase E-1 es descrita por Conde como de gran tamaño, con diámetros de $40-45 \mathrm{~cm}$, pero que sólo excepcionalmente llevan asas, y en estos dos casos, enganchadas por completo.

Sólo hay una producción que fabrique cálatos con asas exentas, éstas son las del tipo B-1. Éstos suelen presentar separaciones verticales de la decoración y tener 'dientes de lobo' en sus bordes. El diámetro externo de su boca va de 28 a $35 \mathrm{~cm}$ y sus pastas son buenas. Su cronología de producción cubre desde finales del s. III hasta mediados del II (cuando sería sustituido por las producciones Fontscaldes) y sus piezas suelen encontrarse en Barcelona, Tarragona y sur de Lérida. Como puede verse la cronología no acaba de coincidir, ni el tamaño es totalmente compatible, así como el que los productos B-1 suelan tener buenas pastas y buena pintura los aleja de los cálatos de la U.E. 1004, de mala pintura que salta con facilidad. Posteriormente, el taller de Fontscaldes hace producciones derivadas de las del taller anterior pero ya con una menor calidad y con diámetros externos de hasta $36 \mathrm{~cm}$. Esto encajaría mejor con nuestra pieza, salvo por el hecho de que estas producciones presentan asas pegadas, no exentas. Respecto a la decoración, la mixta de 'dientes de lobo' y $\mathrm{S}$ es habitual en estas producciones, pero la del cuerpo del vaso es más bien pobre. En los restos conservados no se detectan motivos vegetales como las hojas de hiedra, sino básicamente cabelleras verticales y semicírculos concéntricos; compatibles con decoraciones sencillas del taller de Fontscaldes. Puede concluirse que parece que las piezas de Can Jordi son una producción catalana y que posiblemente corresponda a un taller que recoge la tradición de las piezas B-1 imitándolas con variantes y peor calidad y ya con una decoración simple más propia de Fontscaldes que de las cuidadas producciones previas. Ello nos colocaría cronológicamente precisamente en el tercer cuarto del s. II. Considero que provisionalmente, a falta de un reestudio específico, es lo más probable y que, en todo caso, la homogeneidad de las tres piezas de la U.E. 1004 recomienda considerarlas de un taller cercano.

Por lo tanto tendríamos una inscripción hecha en el sur (o centro) de Cataluña hacia el 135 a.C.
Esta inscripción es leída por Panosa Jbanmí . biurbortel, habiendo tras biuŕ una franja de decoración que lo separa de la palabra siguiente. El signo que Panosa lee $i$ está dañado y no es una lectura segura tal vez se trate de $b a$ seguido de otro o de un separador. Podría especularse con una lectura ban ḿbaŕbiuŕ, con lo que tendríamos un onomástico ḿbaŕ+biuŕ pero no es especialmente probable. En todo caso no puedo aceptar la lectura bo que hace Panosa de un signo que morfológicamente es $t i-3$, con el único propósito de leer un onomástico biuŕbór y sin presentar paralelo alguno en que esa forma pudiera leerse bo. Asimismo, el diferente 'ductus' de la primera ŕ (circular, con dos pasadas en su base) respecto a la segunda (angulosa, con la base hecha en un solo trazo) me hace sospechar no sólo que la franja de decoración sí separe dos palabras o textos, sino incluso que sean de dos autores distintos.

Paleográficamente la inscripción presenta $b i-2$, $t i-3$ y te-1. Debe tenerse en cuenta que es una inscripción pintada, no incisa, y que ya en Liria o Vielle-Toulouse se planteaba la posibilidad de que el estilo de escritura más libre que ofrece el pincel facilitase la creación de formas algo diferentes respecto a las incisas, que son la base de la paleografía. Sin embargo la cronología de ti-3 de post 150 encaja perfectamente con la datación arqueológica de la pieza. El problema radica en la forma te-l que en mi anterior trabajo consideraba que dejaba de utilizarse hacia el 175 , junto con las demás formas circulares de te. Dado lo especial de la pieza el problema es complejo. Si fuese una produccción de Teruel, no habría problema dado que es una zona arcaizante, pero esta posibilidad, si bien menos improbable que la procedencia de Liria, me parece poco verosímil. Restan dos opciones:

La primera es que cabe la posibilidad de que se trate precisamente de un 'ductus' especial debido a que, mientras que la escritura incisa favorece trazos rectos, la pintada favorece los curvos. Desde este punto de vista el signo no sería tanto una perduración sino una recreación tal vez excepcional. Este planteamiento, que puede parecer forzado, tiene en cambio un argumento objetivo a favor. La forma te$l$ (circular con línea diametral vertical) es muy rara, siendo normal el que la línea diametral sea más o menos horizontal (te-2). Desde este punto de vista, es poco probable que el signo de Can Jordi sea una perduración directa.

La segunda es que efectivamente pervivieran formas circulares de te en época tardía, aunque con una frecuencia escasa. Presenta a su favor el que 


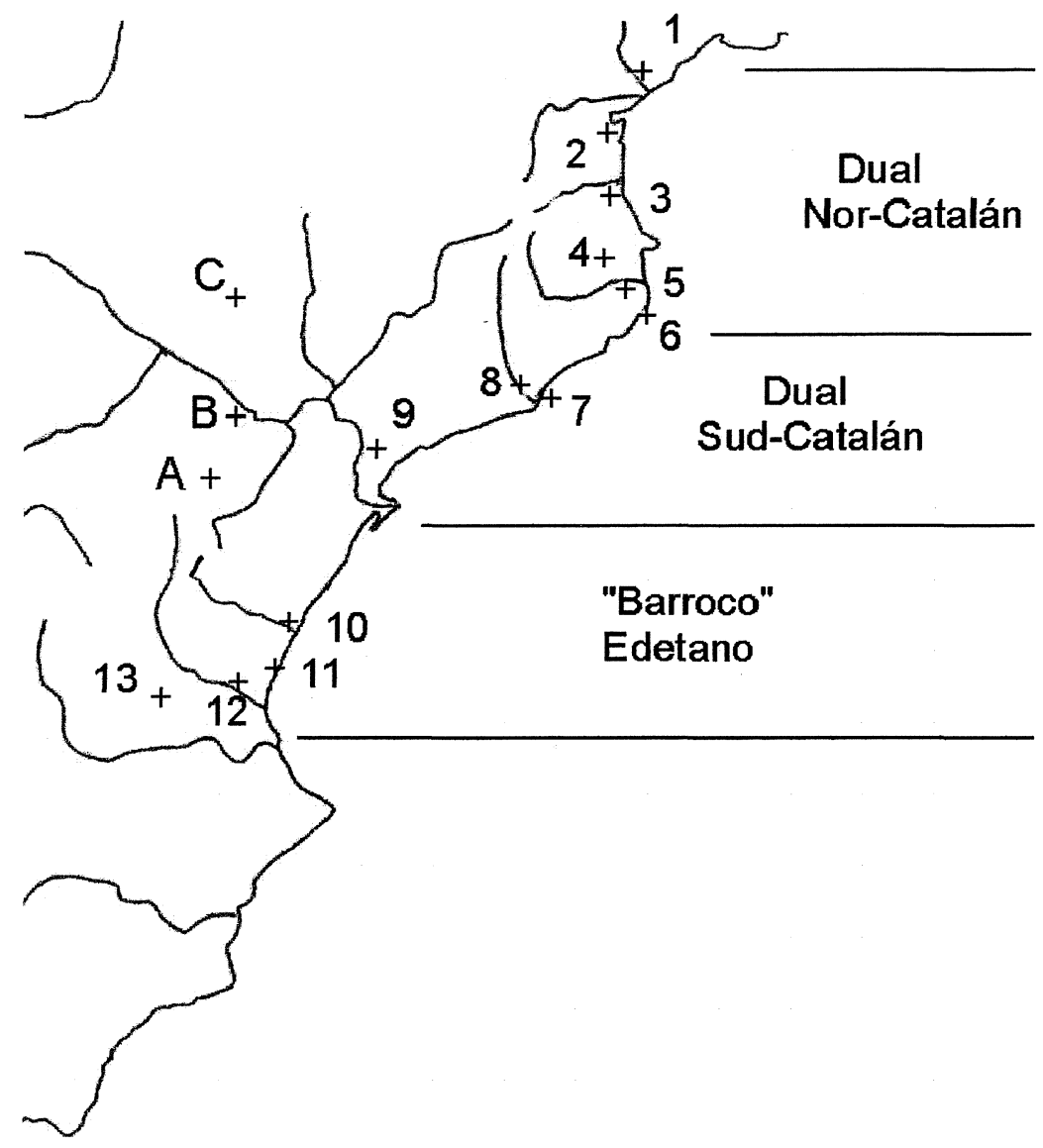

Fig. 1.-Zonas paleográficas del signario ibérico levantino: prerromanas y arcaizantes. Yacimientos significativos con inscripciones de los ss. IV-III: 1. Ensérune; 2. Pech-Maho; 3. Ampurias; 4. Pontós; 5. Ullastret; 6. Palamós; 7. Montjuic; 8. Panya del Moro; 9. Tivissa; 10. Castellón; 11. Sagunto; 12. Liria; 13. Los Villares. Yacimientos con inscripciones tardías arcaizantes: A. Oliete; B. Azaila; C. Huesca. (La mayor parte de las cecas pirenaicas no pueden situarse con exactitud).

resulta un poco extraño esa desaparición de las formas circulares de $t e$ a favor de la forma romboidal te-4 omnipresente en época iberorromana, de estilo lapidario o monetal y antes prácticamente inexistente. Sin embargo, la enorme preponderancia de $t e-4$ es un hecho.

Independientemente de cuál sea la solución correcta el hecho es que ya sea de modo excepcional o poco frecuente pueden encontrarse formas circulares de $t e$ (por lo menos $t e-1$ ) al menos a mediados del s. II. No es que sea una gran novedad, dado que la posibilidad de inscripciones con signos idiosincráticos o mal trazados es algo con lo que siempre hay que contar, pero debe quedar claro que, a la vista de las pruebas, te circular es más un claro indicio de antigüedad de una inscripción que una prueba definitiva. Obviamente, cuantos más signos presente una inscripción, más fundamentada estará una datación.

\section{LA DIVISIÓN EN ZONAS GEOGRÁFICAS SE- GÚN LA PALEOGRAFÍA Y LAS ZONAS ARCAI- ZANTES ${ }^{13}$}

Uno de los objetivos del análisis paleográfico es, aparte del instrumento cronológico, el poder establecer una regionalización de signarios epicóricos o diagrapsos ${ }^{14}$. Dada la gran uniformización que se establece desde el segundo cuarto del s. II en favor de estilos iberorromanos y lo escaso de los testimonios del s. Iv, lo establecible será: de un lado los

13 Un estudio similar, tomando como referencia los signarios meridional y celtibérico y desde un punto de vista histórico geopolítico puede verse en Rodríguez Ramos (en prensa 2). Los aspectos relativos al origen del signario celtibérico, a partir de diversas variantes del levantino, en Rodríguez Ramos (1997b).

14 Diagrapso es a una escritura lo que un dialecto a la lengua. 
grupos territoriales del s. III; de otro las zonas peculiares arcaizantes en fase ya iberorromana.

De acuerdo con la cronología arqueológica y paleográfica de las inscripciones en ibérico levantino disponible en la actualidad, este sistema de escritura cubre en el s. III la costa desde el Hérault en el sur de Francia al paralelo formado por Liria y Los Villares. Dentro de este territorio podemos hacer dos distinciones principales. Al norte, la variante levantina catalana (incluyendo la Cataluña histórica, es decir también la francesa) se distingue por el uso de signos silábicos adicionales para desdoblar algunas oclusivas (se supone que para distinguir sordas de sonoras) en lo que he llamado sistema dual de transcripción de oclusivas. Las inscripciones más meridionales que considero siguen esta norma son la de la pátera y el caliciforme del tesoro de Tivissa (01 y 02). Frente a éstas la más septentrional que lo incumple sería el plomo de Castellón ${ }^{15}$, la frontera ha de estar entre ambos puntos. En principio podemos considerar el Ebro como límite.

Esta distinción del sistema dual desaparece hacia el 200, sin embargo sí que perduran sus divisiones internas. Dentro del grupo catalán podemos hacer dos distinciones en la segunda mitad del s. III. Ésta se puede efectuar por el uso de $a-4$ (en forma de R). Es cierto que hay alguna forma similar a $a-4$ en la ceca de Játiva, así como en un plomo de Alcoy, pero son formas no totalmente desarrolladas probablemente debidas a un conato de evolución paralela. Tampoco hay que tener en cuenta ciertas ánforas itálicas que probablemente son inscripciones latinas AR. El testimonio límite de uso lo tenemos en la fusayola de Palamós-02 en Gerona. Este signo se usa en la zona indiketa de Gerona y en el sur de Francia, aunque sin ser la única variante de $a$ que usan. Recientemente se ha encontrado un sello sobre dolium procedente de Jardí Park (Premià de Dalt, al norte de Barcelona) un signo $a-4$ en la marca laurko (Olesti 1992: 85ss.). Por lo tanto, es posible que el uso de este signo se conociese más al sur de Gerona, pero no conviene olvidar que los dolia son un contenedor económico susceptible de ser transportado, por lo que cabría investigar la posibilidad de que hubiese sido importado desde Gerona. Ésta sería la distinción principal del grupo, que podríamos llamar nor-catalán, pero otros rasgos comunes del mismo serían la perduración clara de $b o-l$ en época iberorromana (aunque sólo se mantendría

15 En mi trabajo anterior sí consideraba que lo cumplía, así como el plomo de Bernabé-01, sin embargo no presentan un uso coherente. De esta manera se explicaría el uso contradictorio que encontramos en el plomo de Castellón (baites junto a baides). como único en el sur de Francia), así como si, como parece, $x$-3 y $x$-4 son el mismo signo, también sería otro rasgo definitorio común.

Pero incluso dentro de este grupo nor-catalán puede hacerse una diferenciación, puesto que pese a la variante de $a$ que los unifica, la zona francesa presenta idiosincracias múltiples que lo distinguen de la zona indiketa. Diferencias que parecen ampliarse con la conquista romana, hecho lógico puesto que el control de Gerona es bastante anterior al del sur de Francia por los romanos. Así ya hemos visto que la forma $b o-2$ está extrañamente ausente en cualquier época en el sur de Francia, por más que es común al resto del levantino hasta el 200 a.C., en que desaparece. Por otra parte, la zona de Gerona se muestra permeable a la innovación iberorromana de bo-3, mientras que esta variante no se documenta en el sur de Francia. Otras diferencias, que se remontan al menos a la segunda mitad del s. III, son el uso de formas complejas de $b u$ (formas 2, 3 y 4) sólo en el sur de Francia, así como su variante también exclusiva $i-2$ (muy similar a la $u$ meridional que dio origen al signo).

Una aplicación interesante de estas diferencias zonales la tenemos en el plomo de Gruissan, procedente de un naufragio de inicios del s. I a.C. ${ }^{16}$. Al proceder la inscripción de un barco, pudo llegar, conjuntamente con el barco, tanto del sur de Francia donde fue hallada, como de cualquier otra zona donde se escribiese en ibérico levantino. Sin embargo, en esta inscripción tenemos presentes tanto la forma $a-4$ que nos delimita que procede de Gerona o el sur de Francia, así como también el signo bo-4 que sí se documenta en Gerona pero no en el sur de Francia. Así pues, parece que este pecio salió de algún puerto gerundense, tal vez de Ampurias; aunque, claro está, nunca puede descartarse que el que hubiese viajado antes fuese el indiketa autor de la inscripción.

Al sur del Ebro, entre Castellón y Liria tenemos un estilo epigráfico en el que también se desdoblan (e incluso ocasionalmente triplican) los signos silábicos de oclusiva, así como se tiende a una caligrafía recargada de signos. Sin embargo para el desdoblamiento de signos no se ha detectado, al menos hasta ahora, ninguna pauta que lo explique como un hecho sistemático más que decorativo. Es el estilo que suelo llamar barroco edetano. Es coherente con este hecho el suponer, como creo prácticamente seguro, el que con la segunda guerra púnica y subsiguiente «pacificación» romana parte de la población

${ }^{16}$ Un estudio paleográfico completo de este plomo en Rodríguez Ramos 1997a: 26. 
edetana pasase a la zona de Teruel (ya como refugiados, ya como tropas auxiliares romanas) y se produjera la adaptación del ibérico levantino para escribir el celtibérico (según el modelo Luzaga). Los celtíberos no adoptaron un sistema para distinguir las oclusivas sordas de las sonoras (sistema que obviamente les hubiese convenido) porque los edetanos no lo usaban ${ }^{17}$.

Con la conquista romana se produce una simplificación del signario, con la desaparición de muchos signos que no se encuentran ya en el segundo cuarto del s. II. Sin embargo, se detectan dos zonas proclives al arcaísmo caligráfico. Una es la zona de Teruel y en general la zona al sur del Ebro en contacto con la escritura celtibérica tipo Luzaga. La segunda parece hallarse en la zona pirenaica, básicamente de Huesca.

La zona pirenaica se conoce muy mal, sólo por unas pocas leyendas monetales como las de bolśken / olsken (CNH: 210), arsaos (CNH: 252) o sesars (CNH: 209). Sin embargo realmente desconocemos si sus usuarios usaban lengua ibérica u otra. Presentan influjos celtibéricos en la forma ke de bolśken, así como la supuesta $e$ de sesars es técnicamente idéntica al signo $x$-4. Respecto a éste podría pensarse en una influencia del grupo nor-catalán pues, no en balde, la equivalente $x$ - 3 parece encontrarse en las inscripciones rupestres de Cerdaña, con lo que tendríamos una conexión pirenaica. Sin embargo, la peculiar $r$ de la ceca arsaos, en forma de R invertida, aboga por una evolución propia fuertemente marcada para esta zona. En todo caso, no deja de ser interesante la aparición ocasional de bo-1, sea marca o signo alfabético, junto al predominante $b o$ 3 , que discutimos supra.

El núcleo de arcaísmo de Teruel puede manifestarse en aquellas muy minoritarias inscripciones de Azaila que presentan signos como $s-3$ y $s-4$ (Rodríguez Ramos 1997a: 18), así como en el caso excepcional de bo- 1 que hemos citado (por más que no sea del todo imposible que en este caso hayamos de contar con una marca numeral). Pero más interesante es el caso de Oliete, yacimiento de la primera mitad del s. I donde el arcaísmo es casi la norma. Tenemos el caso de la bo- 1 en Oliete-01. Pese a que su trazado es un tanto extraño como para ser un bo1 normal, parece que hay que clasificarlo como tal. Muy interesante es la fusayola Oliete-06. En ésta, el único signo que presenta una innovación clara es el

17 Otro argumento sobre la no utilización de sistema dual por los edetanos lo tenemos en la falcata saguntina estudiada en Rodríguez Ramos (1998), datable posiblemente hacia 325-275 a.C. y con una forma nueva de ke que parece el origen de la peculiar $k e-8$. signo $t u$, sin base inferior. No es imposible, aunque tampoco probable, el que se pueda leer este signo como $k a$ y, en toda caso, esta innovación sobre $t u$ es común a la que se encuentra en el celtibérico del tercer bronce de Botorrita. Tampoco es muy antigua la forma de $b a-2$, que nos da un post 275 . Por lo demás la forma ḿ- $1 b$ es una forma clásica (teóricamente la originaria en levantino), la forma $b i-5 c$ es claramente arcaizante (como hemos discutido al tratar de esta forma), mientras que la $e$ de tres trazos es también un rasgo anómalo en esta época. El sentido descendente de sus rasgos horizontales es un rasgo poco frecuente incluso entre las inscripciones ibéricas de los Ss. IV-III: se encuentra con tres trazos en el caliciforme de Tivissa-02 y en una inscripción de Liria (F.13.64), mientras que con cuatro en la mencionada falcata saguntina. Pero sí que es un rasgo normal en la escritura celtibérica tipo Luzaga. Sin embargo, es problemático el tercer signo. Éste se trata de una barra vertical con dos pequeños topes en sus extremos, casi como una I. En principio no hay nada en contra de la lectura que proponen sus editores puesto que con kutubanḿbaŕbianer podríamos tener un onomástico formado por el formante ban y el formante ḿbaŕ. Sin embargo hay dos graves observaciones. La primera es que ya tenemos una forma normal de $b a$ en esta inscripción, lo que hace dudar de que el tercer signo tenga el mismo valor. La segunda es que es raro, si no inusitado, el que el formante ḿbaŕ se encuentre en segunda posición. Sería muy interesante, y desde luego un arcaísmo más, el que este signo I proviniese de la forma $x-3$, con la que guarda una cierta similitud, pero, en principio, no me parece probable.

En todo caso, creo que puede considerarse perfectamente el grupo arcaizante de Teruel teniendo en cuenta dos factores: $1 .^{\circ}$ el aislamiento en Teruel de población edetana desplazada; y $2 .^{\circ}$ el contacto con la escritura celtibérica, la cual conserva muchos rasgos caligráficos del estilo barroco edetano.

\section{APÉNDICE}

Se refieren aquí las equivalencias de las inscripciones respecto a los $M L H$ o a su lugar de edición, así como pormenores de su datación cronológica, sea arqueológica o paleográfica. Algunos aspectos generales, como de yacimientos o de inscripciones concretas, ya fueron detallados en mi anterior artículo y se citan como (1997a: pp.).

Andelos-01: (K.28.1), inscripción sobre mosaico, posiblemente de finales del s. II, una datación prudente es $150-50$. 
Ampurias-06: (C.1.6; 1997a: 26, n. ${ }^{\circ}$ 8) plomo hallado en el Asklepeion, probablemente del estrato de base de la edificación de la segunda mitad del s. II. Datación paleográfica de 175/150-50. Datación probable 175/150-110.

Ampurias-12: (C.1.12), fragmento de cerámica ática. Cron. 400-300.

Ampurias-23: (Sanmartí 1988, Rodríguez Ramos 1997a: 25, n. $\left.^{\circ} 4\right)$, plomo procedente de un estrato arqueológico de 250/225-200. Paleográficamente ca. $220 / 210$

Ampurias-24: (Sanmartí et alii, 1991: n. ${ }^{\circ} 4$ ), base de escifo ático. Cron. 400-300.

Azaila: (1997a: 27) el yacimiento cubre el s. II hasta el 75 a.C., sin embargo el grueso de las inscripciones, si no todas, se enmarca en el lapso 150-75.

Azaila-287: (E.1.289; Beltrán 1995: 71), cerámica común. No puede descartarse que su texto boka no sea una marca ágrafa, aunque boka se encuentra con bo-3 en algunos pondera de Azaila (Beltrán 1995: 23, 34, 53 y 62). Cron.: 200/150-75.

Azaila-318: (E.1.320), ánfora Dressel 1B (Beltrán). Cron. 100-75.

Azaila-320: (E.1.322), ánfora Dressel 1B (Beltrán). Cron. 100-75.

Azaila-369: (E.1.372), peso de mármol procedente de la casa 8 , calle $\mathrm{D}$, de la última fase del poblado. Cron. 100-75.

Azuara-01: (E.3.1), fragmento de dolium. Es probable que sea de los ss. II-I a.C., pero también es probable que sólo sea una serie numeral.

Benidorm-01: (G.8.1), cerámica campaniense B forma Lamboglia 5. Cron. 150-50.

Bernabé-01: (Guérin y Silgo, 1996), lámina de plomo de Castellet de Bernabé procedente de un nivel del poblado datado en ca. 200.

Binéfar-02: (D.12.2) estela funeraria, lo que sugiere un post 200. La paleografía arcaizante de sus signos (be-7, $s$-3 y probablemente $o-5$ ) sugiere un lapso 190-150.

Cabanes-01: (F.5.1) estela funeraria, lo que sugiere una datación de ss. II-I. Presenta los signos $t i$ 4, $\dot{r}-2$ y $s-1$ o $s-2$. La datación sería de 200/150-50.

Can Rodón-02 : (Panosa 1993a: 6.1) protocampaniense del taller de Rosas forma Lamboglia 23. Panosa (1999: 263, 7.2) señala, además de la de Can Rodón, una inscripción idéntica en texto y soporte como procedente de la ciudadela griega de Roses. Se trata de un error de la autora, ambas son la misma inscripción. La producción de la pieza se centra en 275-250, aunque se encontró en la tumba IV junto con campaniense A (Barberá 1969-70: 184), y la amortización parece de finales del s. III. Cron. 275/ 250-200.
Castellón-01: (F.6.1) plomo posiblemente funerario pero sin contexto conocido. Las formas be-8, te-2, te-3 y s-3 le dan un margen de 210-175/150. El uso claro de desdoblamiento de signos de oclusivas apunta a una datación en su margen superior. Cuando aceptaba que este plomo usase el sistema de notación dual lo databa en 210-200 a.C., pero si lo consideramos perteneciente al barroco edetano posiblemente podamos inscribirlo en 210/200-180/ 175. Sería contemporáneo a las últimas producciones de Liria.

Elne-11: (B.9.11) fragmento probablemente de un cálato, pero la pieza no fue localizada para su revisión por Untermann, por lo que no se puede descartar que se trate de otra forma con borde plano. Paleográficamente los signos $a-4$ y $b e-12$ sugieren una datación de 250/225-180. Dado que los primeros cálatos que aparecen en el sur de Francia son de los talleres B-1 a 3 tenemos un post quem de 225 . Cron. 225-180.

El Solaig-01: (F.7.1) su datación paleográfica es de ca. 180 (1997a: 25 n. ${ }^{\circ} 8$ ).

Els Monjos-02: (C.19.2), asa de ánfora, datada según el lapso de ocupación del yacimiento en 12550.

Ensérune: (1997a: 27), estratigráficamente la necrópolis se acaba hacia el 225 , con un inicio hacia el 325. Sobre ella se establece la fase III del poblado, con una cronología de 225-175. Por desgracia el interesantísimo material cerámico de barniz negro de Ensérune sigue sin recibir el estudio a fondo que merecería, lo que da una cierta duda a sus filiaciones.

Ensérune-03: (B.1.3), fondo de cerámica ática de figuras rojas procedente de la necrópolis. Cron. 400300.

Ensérune-16: (B.1.19) crátera proveniente de la necrópolis, posiblemente una Lamboglia-40. Cron. 325-225.

Ensérune-18: (B.1.21), crátera ática similar a la forma Morel 3521c1, datable en 350-300. Procede de la necrópolis. Cron. 325-300.

Ensérune-21: (B.1.24), crátera Lamboglia 40 ática o, más probablemente, protocampaniense procedente de la necrópolis. Cron. 325-225. Paleográficamente la presencia de una forma próxima a $a-5$ sugiere reducir su datación a 275/250-225.

Ensérune-23: (B.1.26), crátera de barniz negro probablemente Lamboglia 40, ática protocampaniense. Tiene reparaciones antiguas, por lo que su amortización funeraria corresponde a un periodo tardío de la pieza. Cron. 325/275-225.

Ensérune-26: (B.1.29), base de cerámica de barniz negro de forma crateroide procedente del pobla- 
do. La calidad de la pieza no parece muy buena y podría ser una campaniense A, ¿tal vez Lamboglia 48 o 49? Paleográficamente presenta los signos $l-2$ y $s-1$ por lo que debe de ser posterior al 225 a.C., posiblemente posterior al 200 a.C.

Ensérune-61: (B.1.64), cerámica campaniense definida por Jannoray como del 'primer estilo' (posiblemente clase A). Juntamente con su paleografía ( $s$ $1, l-2, t e-4)$ puede proponerse una datación de s. II.

Ensérune-124: (B.1.128), si seguimos la descripción de Jannoray para este pequeño fragmento como campaniense del 'primer estilo', la cronología sería de 225-100.

Ensérune-268: (B.1.272), cerámica común. Paleográficamente presenta $r-2 b$ y $l-2$ lo que le otorga un post 200.

Ensérune-271: (B.1.275). Aríbalo de cerámica común, presenta el signo $s-4$ lo que le enmarca en un lapso de 300-175/150.

Ensérune-285: (B.1.289), fragmento de cerámica «gala» rojiza procedente del poblado.

Ensérune-302: (B.1.307), fragmento de cerámica gala rojiza procedente del poblado, presenta la forma $r-2 b$ lo que sugiere un post $225 / 200$.

Ensérune-336 y 337: (B.1.341 y 342), fragmentos de sendas ánforas itálicas, según Jannoray. $\mathrm{Su}$ lapso sería pues de 150/135-50.

Gruissan-01: (Solier y Barbouteau 1988), plomo procedente de un naufragio datado en el primer tercio del s. I a.C. (1997a: 26 n. $\left.{ }^{\circ} 10\right)$.

Liria: El sector alto del poblado es destruido a inicios del s. II. Bonet data el estilo de cerámica pintada tipo Liria en el lapso 250-180. Al mismo corresponden Liria-03: (F.13.3), Liria-16 (F.13.16) y Liria-27 (F.13.27). La presencia de be-11 en Liria-3 lo reduce a 210/200-180 (1997a,25 n. $\left.{ }^{\circ} 6\right)$.

Manises-01: (Lobregat y Rosser 1993) terra sigillata sudgallica Dragendorff 22/23, s. I d.C.

Montlaurés-01: (B.4.1), editado por Untermann como fragmento de cerámica ática, pero sin haberlo podido ver. Cron. 400-300.

Montlaurés-03: (B.4.3), fragmento de cerámica campaniense de baja calidad (225/150-50). Paleográficamente presenta el signo $t i-3$ por lo que sería posterior al 150 .

Oliete: yacimiento con una única fase del primer tercio del s. I a.C. en la que sus excavadores datan todas sus piezas. Oliete-01 (E.5.1).

Oliete-06: (Oliete : $27 \mathrm{y} \mathrm{n}^{\circ}{ }^{\circ}$ 90) fusayola en contexto de 100-65.

Oliete-08: (Oliete : n. ${ }^{\circ}$ 92) pesa de telar en contexto 100-65.

Orleyl-01: (F.9.1), plomo de procedencia atribuida. Paleográficamente (signos $l-2, s-1$ y una varian- te de $b e-14)$ parece datable en $225-180$, probablemente a inicios del s. II.

Orleyl-05-07: (F.9.5-7), conjunto de tres plomos procedente de la tumba II y con ajuar ático cuyo ejemplar más moderno sería de ca. 325 . Paleográficamente es poco probable datarlo en el s. IV por lo que parece que el material ático ha sido amortizado muy tarde. Lapso posible: $275-225$ (1997a: 24 n. 3).

Osséja-02: (Campmajó y Untermann 1993: n. ${ }^{\circ} 6$, pp. 507-509), inscripción rupestre. Como todas las demás de la Cerdaña, se le supone posterior a finales del s. III. Estas inscripciones son problemáticas, pero paleográficamente, por la forma $b e-11$, puede sugerirse un lapso de 210-175.

Palamós-01 (C.4.1), plomo aparecido en un contexto de 300-250 (1997a: 24, n. ${ }^{\circ}$ 2).

Palamós-02 (C.4.2), fusayola bitroncocónica. Presenta los signos $b e-7, a-4,-2$ y $s-3$ por lo que la datación paleográfica es de 190-150.

Pech-Maho: los plomos Pech-Maho 34 a 38 (Pech-Maho I a V: Solier 1979 y Solier y Barbouteau 1988) corresponden a una fase del poblado de la segunda mitad del s. III, probablemente a su momento final en el último cuarto. La paleografía también indica un lapso de 225-200 (1997a: 25, n. ${ }^{\circ}$ 5 y 28).

Pech-Maho-02: (B.7.2), base de cerámica ática, probablemente un escifo. Cron. 400-300.

Por_Granada-01: (Untermann 1998), plomo supuestamente aparecido en Pinos Puente. Presenta el signo te-2 (excepcionalmente también te-1), $s$-3 y 4 , una variante evolucionada de $b e-11$. Encajaría en el periodo 210-180/175.

Por_Tarragona-01: (C.0.1) plomo sin contexto. Sus signos (s-1 y a-5) dan un post quem de 225/200.

Por_Tarragona-02: (C.0.2), plomo sin contexto. Los signos $s-5, s-3$ y $l-2$ le enmarcan en un lapso 225-150.

Sagunto-26: (F.11.26), fragmento de escifo de cerámica ática producido a inicios del s. IV. Cron. 400-300.

Sagunto-29: (F.11.31), plomo sin contexto conocido, por los signos $a-5$ y $s-3$ puede proponerse un lapso de 225-150.

Sidamunt-01: (D.7.1) cerámica ática de barniz negro Lamboglia 21 que por sus características formales (decoración y tipo de pie) ha de ser posterior al 375 (Barberà 1964-65: 142). Como se ha indicado, existe la posibilidad de considerar que la inscripción sea muy posterior.

Solsona-03: (D.5.3), fragmento de campaniense. Cura indica que este yacimiento se inicia a finales del s. II. Cron. 120-50. 
Tivissa-01 y 02 (C.21.1 y 2) pátera de plata umbilicada con rica decoración iconográfica y vaso caliciforme de plata procedente de un tesoro, probablemente una ocultación de época de Catón (195); lo que le da un ante quem. El uso de ba-2 y de notación dual sugiere paleográficamente un lapso de 275-200.

Ullastret-03: (C.2.3), sin contexto arqueológico válido, su datación paleográfica apunta a 350-300 (1997a: 24, n. ${ }^{\circ}$ 1).

Ullastret-08: (C.2.8), figura animal de cerámica (comúnmente denominada 'rhyton de Ullastret'). Ha de ser anterior al 190, fecha de abandono del poblado. Su caligrafía es muy deficiente con signos mal trazados y poco visibles. Al no apreciarse uso del sistema dual es posible que corresponda al momento final del poblado (200-190), pero las dificultades de lectura de los signos aconsejan la prudencia.

Ullastret-13: (C.2.13), base de cerámica ática. Cron. 400-300.

Ullastret-23: (C.2.23), fragmento de cerámica ática. Cron. 400-300.

Vieille-Toulouse: (Vidal y Magnol 1983) conjunto de ánforas grecoitálicas (republicana I de Benoit), probablemente de la clase LWe (175-100). El material acompañante supone una amortización hacia el 170-130 a.C. (1997a : 29) ${ }^{18}$.

Villares-01 y 02: (F.17.1 y 2), dos plomos sin contexto. Paleográficamente (signos be-9, te-2, te-3, $s-3, s-4, o-3$ y $b a-2$ ) datables en 275/250-200.

Yátova-01-03: (F.20.1-3; 1997a: 26 n. ${ }^{\circ}$ 9) conjunto de tres plomos datable paleográficamente en 150-50.

\section{BIBLIOGRAFÍA}

BARBERÀ, J., 1964-65: «La cerámica barnizada de negro del poblado ilergeta del Tossal de les Tenalles de Sidamunt (Lérida)», Ampurias XXVIXXVII, 135-163.

BARBERÀ, J., 1969-70: «La necrópolis ibérica de Cabrera de Mar (Excavación 1968-1969)», Ampurias XXXI-XXXII, 169-189.

BELTRÁn LloRIs, M., 1976: Arqueología e historia de las ciudades antiguas del Cabezo de Alcalá de Azaila (Teruel), Zaragoza.

Beltrán, M., 1990: Guía de la cerámica romana, Zaragoza.

BeltrÁn Lloris, M., 1995: Azaila. Nuevas aportaciones deducidas de la documentación inédita de Juan Cabré Aguiló, Zaragoza.

${ }_{18}$ Panosa (1993b: 98 y 1999: 166) las clasifica como Dressel 1A y $1 \mathrm{~B}$ del segundo cuarto del s. II.
Bonet Rosado, H., 1995: El Tossal de Sant Miquel de Llíria. La antigua Edeta y su territorio, Valencia.

Conde, M. ${ }^{a}$ J., 1991: «Les produccions de kálathoi d'Empúries i la seva difusió mediterrània (segles II-I a. C.)», Cypsela IX, 141-168.

Conde i Berdós, M. ${ }^{a}$ J., 1992: «Una producció ceràmica característica del món ibèric tardà: el kalathos 'barret de copa'», Fonaments 8, 117169.

Cura i Morera, M., 1985: «Les ceràmiques de vernís negre de Can Sotaterra a Solsona i l'estratigrafia comparada dels jaciments pre-romans del Solsonés», Faventia 7/2, 105-113.

Cura i Morera, M., 1993: «Nous grafits ibèricas en el Molí d'Espígol (Tornabous) i la cronologia de l'escriptura ibèrica a l'interior de Catalunya», Gala 2, 219-225.

GuérIN, P. y SiLGO, L., 1996: «Inscripción ibérica sobre plomo de Castellet de Bernabé (Llíria, Valencia)», RAP 6, 199-206.

JANNORAY, J., 1955: Ensérune. Contribution à l'étude des civilisations prérromaines de la Gaule Meridionale, Paris.

Lafuente I Revuelto, A., 1992: «La producció de ceràmica ibèrica del taller de Fontscaldes (Valls, Alt Camp)», en Societat Catalana d'Arqueologia (ed.) Les ceràmiques de tècnica ibèrica a la Catalunya Romana, Barcelona, 47-77.

Lobregat, M. y Rosser LimiñanA, P., 1993: «Un fragmento cerámico con grafitos bilingües, y la pervivencia del sustrato ibérico en la ciudad romana del Tossal de Manises (Albufereta, Alicante)», LQNT 1, 119-135.

Olesti i Vila, O., 1995: El territori del Maresme en època republicana (s. III - I a.C.). Estudi d'Arqueomorfologia i Història, Mataró.

Oliete hace dos mil años, (catálogo de exposición), 1990 Teruel.

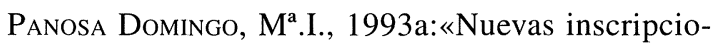
nes ibéricas de Cataluña», Complutum 4, 175222.

Panosa Domingo, M. ${ }^{\text {a }}$ I., 1993b: «Aproche comparée de l'écriture ibérique en Languedoc-Roussillon et en Catalogne», DAM 16, 93-103.

Panosa, M. ${ }^{a}$ I., 1996: «Elementos sobre la fase de bilingüismo y latinización de la población ibérica», en Villar, F. y d'Encarnação, J. (eds) La Hispania Prerromana, Salamanca, 217-246.

PANOSA, M. ${ }^{a}$ I., 1999: La escritura ibérica en Cataluña y su contexto socioeconómico (siglos V-I a. C.), Vitoria-Gasteiz.

Prevosti, M., ArXé, J. y Caballé, A., 1995: Autopistas i Arqueologia. Memòria de les excavacio- 
ns en la prolongació de l'autopista A-19, Barcelona.

Rodríguez Ramos, J., 1994: «Liria XIIC: ¿un kálathos ibérico dedicado a Proserpina?», Faventia $16 / 2,65-81$.

Rodríguez RAmos, J., 1997: «Primeras observaciones para una datación paleográfica de la escritura ibérica», $A E \operatorname{spA}$ 70, 13-30.

Rodríguez Ramos, J., 1997b: «Sobre el origen de la escritura celtibérica», Kalathos 16, 189-197.

Rodríguez RAmOS, J., 1998: «Sobre la lectura y la paleografía de la inscripción de la falcata saguntina MPV 314», Pyrenae 29, pp. 227-230.

Rodríguez Ramos, J. (en prensa 1): Análisis de Epigrafía Íbera, 'Baspedas' 1; (edición de tesis doctoral, dirigida por F. Gracia Alonso, revisada a 44-1997), Barcelona.

Rodríguez RAmos, J. (en prensa 2): «La cultura ibérica desde la perspectiva de la epigrafía».

SANMARTí Grego, E., 1978: La cerámica campaniense de Emporion y Rhode, Barcelona.

SAnMARTí-Grego, E., 1988: «Una carta en lengua ibérica escrita sobre plomo, procedente de Emporion», RAN 21, pp. 95-113.

Sanmartí, E.; Castanyer, P.; Santos, M. y TremoleDA, J., 1991: «Testimonios epigráficos de la presencia de población indígena en el interior de Emporion», Huelva Arqueológica XIII,2, 205-214.

SOLIER, Y., 1979: «Découverte d'inscriptions sur plombs en écriture ibérique dans un entrepôt de Pech-Maho (Sigean)», RAN XII, 55-123.
Solier, Y. y Barbouteau, H., 1988: «Découverte de nouveaux plombs, inscrit en ibère, dans la région de Narbonne», RAN 21, pp. 61 - 94.

Untermann, J., 1975: Monumenta Linguarum Hispanicarum Bd. I: Die Münzlegenden, Wiesbaden.

UntermanN, J., 1980: Monumenta Linguarum Hispanicarum Bd. II: Die Inschriften in iberischer Schrift aus Südfrankreich, Wiesbaden.

UNTERMANN, J., 1989: «Nova inscripció ibèrica sobre plom, procedent del país dels Ilergetes», Acta Numismática 19, 39-44.

Untermann, J., 1990: Monumenta Linguarum Hispanicarum Bd.III : Die iberischen Inschriften aus Spanien (2 vols.), Wiesbaden.

UntermanN, J., 1997: Monumenta Linguarum Hispanicarum. Band IV. Die tartessischen, keltiberischen und lusitanischen Inschriften, Wiesbaden.

UNTERMANN, J., 1998: «Comentario sobre una lámina de plomo con inscripción ibérica de la colección D. Ricardo Marsal, Madrid», Habis 29, 7-21.

VelazA, J., 1994: «Sobre dos plomos con escritura ibérica: una revisión y una noticia», Epigraphica $56,9-28$.

VidAL, M. y MaGNOL, J. P., 1983: «Les inscriptions peintes en caractères ibériques de Vieille-Toulouse (Haute-Garonne)», RAN XVI, 1-28.

Villaronga, Ll., 1994: Corpus Nummum Hispaniae ante Augusti Aetatem, Madrid.

Villaronga, Ll., 1998: Les dracmes ibèriques $i$ llurs divisors, Barcelona. 